\title{
Avaliação do quadro clínico e perfil bioquímico de bovinos durante indução e tratamento de hipocalcemia
}

\author{
Assessment of clinical and biochemical profile of cattle during induction and treatment of \\ hypocalcemia
}

\author{
Raimundo Alves BARRÊTO JÚNIOR ${ }^{1}$; Antonio Humberto Hamad MINERVINO²; Frederico \\ Augusto Mazzocca Lopes RODRIGUES²; Enoch Brandão de Souza MEIRA JÚNIOR ${ }^{2}$; Rodrigo N. F. \\ FERREIRA $^{2}$; Alessandra Silva LIMA ${ }^{3}$; Clara Satsuki MORI ${ }^{2}$; Isabela de Oliveira BARROS ${ }^{1}$; Enrico \\ Lippi ORTOLANI ${ }^{2}$
}

${ }^{1}$ Universidade Federal Rural do Semi-Árido, Mossoró-RN, Brasil

${ }^{2}$ Departamento de Clínica Médica, Faculdade de Medicina Veterinária e Zootecnia da Universidade de São Paulo, São Paulo-SP, Brasil

${ }^{3}$ Médica Veterinária. Coordenadora de Pesquisa e Desenvolvimento, Vallée S.A., São Paulo-SP, Brasil

\begin{abstract}
Resumo
O presente trabalho objetivou estudar o quadro sintomatológico, algumas variáveis bioquímicas e a resposta ao tratamento com cálcio de bovinos com hipocalcemia induzida experimentalmente. Foram utilizadas 12 novilhas distribuídas nos grupos controle $(n=5)$ e tratado $(n=7)$. Foi infundida solução de EDTA a 5\% até o animal apresentar sinais clínicos de hipocalcemia, quando então era iniciado o tratamento com solução contendo cálcio, fósforo, magnésio e glicose, na dose de $1 \mathrm{~mL} / \mathrm{kg} / \mathrm{PV}$, em 30 minutos, enquanto que o grupo controle recebia apenas solução fisiológica na mesma dose. Exame clínico e coleta de amostras sanguíneas foram realizados nos tempos T0 (basal), T1 (Fase I, caracterizada por tremores musculares), T2 (ao final da infusão com EDTA), T3 (ao final do tratamento) e T4 (24 horas após o término do experimento). Todas as novilhas mostraram diminuição temporária da concentração de cálcio total e livre, fósforo, e apresentaram quadro clássico de hipocalcemia. A taquicardia, a hipofonese e a atonia ruminal desapareceram no decorrer do tratamento, sendo observado aumento no cálcio livre e total e fósforo. O medicamento usado no tratamento dos animais foi eficaz na recuperação do quadro clínico de hipocalcemia dentro de 30 minutos, promovendo retorno das principais variáveis do perfil bioquímico aos valores basais.
\end{abstract}

Palavras-chave: Cálcio. Fósforo. Tratamento. Hipocalcemia. EDTA.

\begin{abstract}
The present work aims to study the clinical picture, biochemical profile and treatment response in cattle with induced hypocalcaemia. Were utilized 12 heifers randomly distributed in treated $(n=7)$ and control $(n=5)$ groups. The induction model was carried on by continuous EDTA infusion into jugular vein until the animals present clinical signs of hypocalcaemia. After that, the treated group received a calcium $(\mathrm{Ca})$ solution enriched with phosphorus, magnesium and glucose with a dose of $1 \mathrm{~mL} / \mathrm{kg} / \mathrm{BW}$ in 30 minutes, meanwhile, the control group was treated with the same dose of physiologic solution. Clinical examination were performed and blood samples were obtained in times T0 (basal time), T1 (beginning of hypocalcaemia); T2 (end of EDTA infusion); T3 (end of treatment) and T4 (24 hours after the induction). All the heifers present temporary blood calcium and phosphorus reduction and demonstrated classical clinical picture of hypocalcaemia. The treated group present full clinical recovery and blood calcium and phosphorus increase. Most evident clinical signs were increasing heart beat, hypophonesis and rumenal atony. Those symptoms were reversed after calcium treatment. The solution used for treatment was efficient on clinical recovery within thirty minutes, promoting the return to basal levels of the most of biochemical's variables.
\end{abstract}

Key-words: Calcium. Phosphorus. Treatment. Hypocalcaemia. EDTA.

\section{Introdução}

O cálcio $(\mathrm{Ca})$ é um macroelemento essencial na formação do esqueleto, coagulação do sangue, regulação do ritmo cardíaco, excitabilidade neuromuscular, ativação enzimática, permeabilidade de membranas entre outras importantes funções ${ }^{1,2}$. O organismo animal tem um sistema de regulação muito controlado

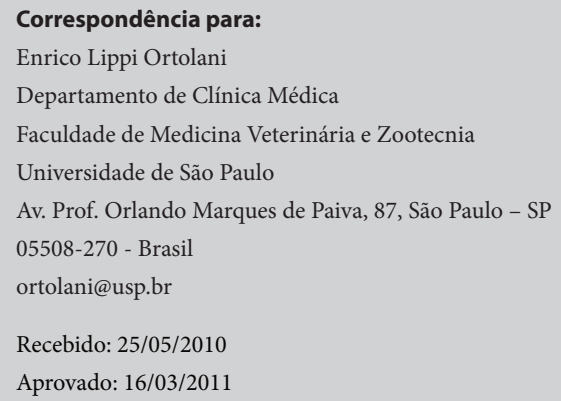


das concentrações de cálcio ionizável no sangue, as quais têm que permanecer dentro de patamares restritos, ou graves consequências poderão advir tanto da hipo como da hipercalcemia ${ }^{3}$.

No período que antecede ao parto existe uma tendência para que as vacas apresentem certa dificuldade para manter a homeostase da calcemia, em particular, a fração do cálcio ionizável. Tanto os níveis de absorção intestinal como reabsorção óssea estão diminuídos nos últimos sete dias antes e dois dias após o parto. Além disso, a demanda de cálcio está aumentada, pois o colostro produzido contém o dobro das quantidades de cálcio $(2,3 \mathrm{~g} / \mathrm{L})$ que o leite comum $(1,1 \mathrm{~g} / \mathrm{L})^{4}$. Torna-se essencial neste período que os mecanismos de reabsorção óssea estejam adequados para manter a calcemia. Caso isto não ocorra, o animal retira o cálcio da fração ionizável do sangue para produção do colostro ocasionando o quadro clínico de hipocalcemia ${ }^{5}$.

A hipocalcemia da vaca parturiente é uma enfermidade cosmopolita, tendo incidência elevada nos países nórdicos (11\%) e relativamente alta no Estado de São Paulo, Brasil $(4,25 \%)^{4}$. Recente trabalho realizado no Rio Grandes do Sul mostra que esta enfermidade corresponde a 69,98\% das doenças metabólicas diagnosticadas, causando perdas anuais bastante elevadas ${ }^{6}$.

O diagnóstico da hipocalcemia da vaca parturiente poder ser firmado por meio de dosagens do cálcio sérico total e ionizável, ou do diagnóstico clínico da enfermidade seguido da resposta ao tratamento ${ }^{3}$. Menores valores séricos de cálcio e fósforo foram observados nas vacas hipocalcêmicas $(1,55 \mathrm{mMol} / \mathrm{L}$ e 1,04 $\mathrm{mMol} / \mathrm{L})$ quando comparadas com vacas hígidas $(2,2$ $\mathrm{mMol} / \mathrm{L}$ e $1,65 \mathrm{mMol} / \mathrm{L})^{4}$. Valores normais e diminuídos de fósforo plasmático têm sido observados por vários autores em casos espontâneos e experimentais desta enfermidade ${ }^{7}$.

Deste modo o presente estudo objetivou estudar a eficácia de uma solução rica em cálcio (oriundo de três fontes diferentes), fósforo e magnésio no tratamento da hipocalcemia em bovinos, avaliando a sin- tomatologia e o perfil bioquímico durante a indução e a recuperação dos animais.

\section{Material e Método}

Foram utilizadas 12 fêmeas bovinas hígidas e não prenhes, da raça holandesa preta e branca, com peso variando entre 300 e $360 \mathrm{~kg}$ e idade entre dois a três anos, procedentes de um único rebanho do município de Pirassununga, Estado de São Paulo.

Um mês antes do início do período experimental os animais foram desverminados, vacinados contra clostridioses. Foi realizado banho com carrapaticida e aplicação de polivitamínico mineral. O estudo clínico foi realizado nas dependências do Hospital Veterinário da FMVZ-USP, campus de Pirassununga, Estado de São Paulo. Os animais foram alocados em três piquetes de $200 \mathrm{~m}^{2}$, permanecendo quatro animais em cada piquete. Durante o período de adaptação e no decorrer do experimento foi oferecida ração total, calculada em relação a 2,7\% do peso vivo, composta de $75 \%$ da matéria seca (MS) de feno de capim Coastcross e $25 \%$ da MS de ração concentrada comercial, fornecida duas vezes ao dia.

O delineamento experimental foi inteiramente casualizado, com distribuição dos animais em dois grupos, o controle $(\mathrm{n}=5)$ e o tratado com cálcio $(\mathrm{n}=$ 7). O modelo de indução de hipocalcemia seguiu o descrito por Smith e Brown ${ }^{8}$ por meio de infusão venosa de solução de ácido etilenodiaminotetracético dissódico (EDTA) a 5\%, com pH ajustado para 7,4. com velocidade de $220 \mathrm{~mL} /$ hora. No momento que o animal apresentava os sinais clínicos clássicos de hipocalcemia, ou seja, decúbito esternal com atitude de autoauscultação ou decúbito lateral, a infusão era imediatamente encerrada.

A partir do quadro de hipocalcemia estabelecido, os bovinos do grupo tratado recebiam uma solução contendo 2,44 g de cálcio total oriundo de três fontes (Gluconato de Cálcio monoidratado, Lactato de Cálcio pentaidratado e D-Sacatato de Cálcio tetraidrata- 
do), 0,185 g magnésio, 0,472 $\mathrm{g}$ de fósforo (Hipofosfito de magnésio hexaidratado) e $5 \mathrm{~g}$ de Dextrose anidra para cada $100 \mathrm{~mL}$, na dose de $1 \mathrm{~mL} / \mathrm{kg} / \mathrm{PV}$, durante 30 minutos, enquanto que o grupo controle recebia a mesma dose de solução fisiológica. Após o período de acompanhamento de 30 minutos, todos os animais do grupo controle foram tratados com a mesma dose da solução usada no grupo tratado, a fim de promover a recuperação dos animais.

Nos momentos T0 - Tempo zero (basal, antes do início da infusão de EDTA); T1 - Fase I da hipocalcemia, caracterizada por tremores musculares; T2 - Fase II da hipocalcemia, caracterizada por decúbito esternal com atitude de autoauscultação ou decúbito lateral; T3 - Após o término do tratamento, 30 minutos após o término da infusão de EDTA e T4 - 24 horas após o término do experimento; foram realizados exames clínicos e coletas de sangue.

O exame clínico seguiu as recomendações de Feitosa ${ }^{9}$, com avaliação da frequência cardíaca (FC), frequência respiratória (FR), movimentos ruminais (MR), temperatura retal (TR) e tempo de preenchimento capilar (TPC). As coletas de sangue foram realizadas por meio de venipunctura da jugular externa utilizando-se tubos de coleta a vácuo contendo ou não anticoagulante (Fluoreto de Sódio) para obtenção de soro e plasma, respectivamente. No soro foram feitas as determinações da proteína total, albumina, gama glutamiltransferase (GGT), aspartato aminotransferase (AST), creatina quinase $(\mathrm{CK})$, cálcio $(\mathrm{Ca})$, magnésio $(\mathrm{Mg})$ e fósforo (P) enquanto no plasma analisou-se a glicose. Todas as análises foram realizadas em analisador bioquímico automático, utilizando-se kits comerciais. Os resultados das atividades das enzimas foram determinados em $30^{\circ} \mathrm{C}$, segundo recomendação de Kaneko, Harvey e Bruss ${ }^{10} \mathrm{O}$ cálcio livre ou ionizável foi estimado utilizando-se fórmula clássica, descrita por Brito ${ }^{11}$.

As análises estatísticas foram realizadas intragrupo, considerando o tempo zero como controle dentro dos grupos tratado e controle; e entre grupos, comparando os dois grupos em cada um dos momentos avaliados. Todos os dados foram testados quanto à sua distribuição, utilizando-se o teste de Kolmogorov-Smirnov. Os dados que tiveram distribuição normal foram submetidos à análise de variância (teste F), com os grupos comparados pelo teste de Tukey ${ }^{12}$. Análises de regressão e seus respectivos coeficientes de determinação foram utilizados para a verificação da relação de pares variáveis. Foi adotado grau de significância mínimo de $5 \%$ e as análises estatísticas foram realizadas com auxílio de software estatístico ${ }^{13}$.

\section{Resultados e Discussão}

No decorrer da infusão foi constatado, na maioria dos animais, movimento cinético involuntário de língua, lambendo o muflo e a narina, estalido dos lábios, sucção de ar entre os dentes, protrusão de língua, diminuto grau de sialorreia e movimentos de mastigação meríssica. Nesse momento, os animais se tornavam inquietos e desenvolveram rigidez dos membros pélvicos.

Em seguida, os animais apresentavam apatia, incoordenação motora e andar cambaleante, seguidos de decúbito esternal e por fim de decúbito lateral. A maioria das novilhas, antes de permanecerem em decúbito lateral, manteve atitude de autoauscultação. Neste momento, o alimento usualmente oferecido foi recusado pelos animais. Com o aprofundar da infusão, a depressão na consciência aumentou e as novilhas passaram a demonstrar estado de sonolência. A atonia ruminal neste estágio era marcante e muitas novilhas apresentaram quadro de meteorismo gasoso ruminal. Neste estágio, a constatação de anúria e ausência de defecação foram frequentes.

A maioria dos animais apresentou quadro de midríase e perda de reflexo anal. A temperatura cutânea nas extremidades estava aparentemente diminuída. A taquicardia foi evidente associada a quadro de hipofonese.

Após o término da injeção de EDTA, os animais do grupo controle, durante a infusão de solução fisiológica no decorrer de 30 minutos, continuaram a apresen- 
tar quadro de depressão no estado geral, apatia, falta de resposta aos estímulos tácteis e sonoros, midríase e perda de reflexo anal. Três das cinco novilhas se mantiveram em decúbito lateral.

Quadro de meteorismo gasoso leve foi evidenciado em algumas novilhas. Outros sinais clínicos observados com menor frequência foram: movimentos de pedalagem, mioclonia e trismo, acentuada dispneia, seguida ou não de apneia, e respiração ruidosa.

Os resultados da frequência cardíaca e respiratória são observados na tabela 1 , enquanto que da atividade ruminal e do tempo de preenchimento capilar encontram-se na tabela 2. Não foram observadas diferenças significativas intra ou entre grupos para as variáveis frequência respiratória e temperatura retal, permanecendo dentro dos valores normais em todos os momentos.
O quadro clínico de hipocalcemia induzido pelo EDTA provocou sintomatologia clássica nas novilhas de ambos os grupos (tratado e controle), com o surgimento de duas das três principais fases da enfermidade, ou seja: de excitabilidade e tremores musculares (Fase I); depressão do estado geral, paresia muscular, decúbito esternal e atitude de autoauscultação (Fase II). Alguns animais também chegaram a manifestar sintomas da terceira e derradeira fase com decúbito lateral, intenso quadro de depressão e marcante taquicardia (mais de 100 batimentos por minuto) e hipofonese.

A patogenia destes principais sintomas descritos acima está intimamente ligada a intensidade da hipocalcemia presente no momento da indução ${ }^{3,14}$. A ligeira hipocalcemia inicial desnecadeia um quadro de excitação, tetania, com a manutenção da consciência e com o animal ainda em estação. Com a intensificação da hi-

Tabela 1 - Valores médios e desvio padrão (DP) da frequência cardíaca (FC) e frequência respiratória (FR) dos bovinos dos grupos tratado e controle nos diferentes tempos - Pirassununga - SP

\begin{tabular}{|c|c|c|c|c|c|c|c|c|}
\hline \multirow[t]{3}{*}{ Tempos } & \multicolumn{4}{|c|}{ Grupo Tratamento } & \multicolumn{4}{|c|}{ Grupo Controle } \\
\hline & \multicolumn{2}{|c|}{ FC } & \multicolumn{2}{|c|}{ FR } & \multicolumn{2}{|c|}{ FC } & \multicolumn{2}{|c|}{ FR } \\
\hline & Média & DP & Média & DP & Média & DP & Média & DP \\
\hline T 0 & $64,0^{\mathrm{B}}$ & 2,3 & 30,3 & 6,1 & $57,6^{\mathrm{C}}$ & 5,4 & 36,0 & 4,0 \\
\hline T 1 & $86,9^{\mathrm{A}}$ & 21,0 & 33,7 & 13,5 & $74,4^{\text {в }}$ & 10,0 & 35,2 & 14,8 \\
\hline T 2 & $90,0^{\mathrm{A}}$ & 20,9 & 36,3 & 15,2 & $96,8^{A}$ & 9,6 & 43,2 & 20,1 \\
\hline T 3 & $63,4^{\text {B }}$ & 7,1 & 29,1 & 6,4 & $56,4^{\mathrm{C}}$ & 5,4 & 39,6 & 13,6 \\
\hline T 4 & $64,0^{\mathrm{B}}$ & 2,3 & 31,1 & 4,3 & $59,6^{\mathrm{cb}}$ & 1,7 & 35,2 & 3,4 \\
\hline$P$ & \multicolumn{2}{|c|}{0,001} & \multicolumn{2}{|c|}{0,687} & \multicolumn{2}{|c|}{0,001} & \multicolumn{2}{|c|}{0,828} \\
\hline
\end{tabular}

Letras maiúsculas distintas nas colunas indicam diferenças significativas entre os tempos para cada variável. Não foram observadas diferenças entre grupos para as variáveis FC e FR

Tabela 2 - Valores médios e desvio padrão dos movimentos ruminais (MR) e do tempo de preenchimento capilar (TPC) dos bovinos dos grupos tratado e controle nos diferentes tempos - Pirassununga - SP

\begin{tabular}{|c|c|c|c|c|c|c|c|c|c|c|}
\hline \multirow[t]{3}{*}{ Tempos } & \multicolumn{4}{|c|}{ Grupo Tratamento } & \multicolumn{4}{|c|}{ Grupo Controle } & \multirow{3}{*}{$\begin{array}{c}P \\
\text { MR }\end{array}$} & \multirow{3}{*}{$\begin{array}{c}P \\
\text { TPC }\end{array}$} \\
\hline & \multicolumn{2}{|c|}{$\begin{array}{c}\text { MR } \\
\left(\mathrm{mov} / \mathrm{3}^{\prime}\right)\end{array}$} & \multicolumn{2}{|c|}{$\begin{array}{c}\text { TPC } \\
\text { (seg.) }\end{array}$} & \multicolumn{2}{|c|}{$\begin{array}{c}\text { MR } \\
\left(\operatorname{mov} / 3^{\prime}\right)\end{array}$} & \multicolumn{2}{|c|}{$\begin{array}{c}\text { TPC } \\
\text { (seg.) }\end{array}$} & & \\
\hline & Média & DP & Média & DP & Média & DP & Média & DP & & \\
\hline T 0 & $2,6^{\mathrm{A}}$ & 0,8 & $2,4^{\mathrm{B}}$ & 0,5 & $2,4^{\mathrm{A}}$ & 0,6 & $2,8^{\mathrm{B}}$ & 0,4 & 0,685 & 0,235 \\
\hline T 1 & $0,4^{\mathrm{C}}$ & 0,5 & $3,1^{\mathrm{A}}$ & 0,4 & $0,0^{\text {в }}$ & 0,0 & $3,6^{\mathrm{AB}}$ & 0,9 & 0,108 & 0,248 \\
\hline T 2 & $0,0^{\mathrm{C}}$ & 0,0 & $3,0^{\mathrm{Ab}}$ & 0,6 & $0,0^{\mathrm{B}}$ & 0,0 & $4,0^{\mathrm{Aa}}$ & 0,7 & - & 0,022 \\
\hline T 3 & $1,4^{\mathrm{Ba}}$ & 0,5 & $2,6^{\mathrm{AB} \mathrm{b}}$ & 0,5 & $0,4^{\mathrm{B} \mathrm{b}}$ & 0,5 & $4,0^{\mathrm{Aa}}$ & 0,7 & 0,009 & 0,003 \\
\hline T 4 & $2,9^{\mathrm{A}}$ & 0,4 & $2,4^{\mathrm{B}}$ & 0,5 & $2,4^{\mathrm{A}}$ & 0,4 & $2,8^{\mathrm{B}}$ & 0,4 & 0,599 & 0,235 \\
\hline$P$ & \multicolumn{2}{|c|}{0,001} & \multicolumn{2}{|c|}{0,036} & \multicolumn{2}{|c|}{0,001} & \multicolumn{2}{|c|}{0,013} & - & - \\
\hline
\end{tabular}

Letras minúsculas distintas nas linhas indicam diferenças significativas entre os grupos. Letras maiúsculas distintas nas colunas indicam diferenças significativas entre os tempos 
pocalcemia surge a diminuição da força de contração da musculatura cardíaca, levando a concomitante menor eficiência do débito cardíaco. Tal situação desencadeia uma resposta animal de compensação por meio do aumento de frequência cardíaca, embora esta esteja acompanhada de hipofonese.

A diminuição da pressão arterial faz com que a temperatura cutânea das extremidades esteja reduzida e ocorra aumento no tempo de preenchimento capilar, em especial verificado no grupo controle após trinta minutos de infusão de solução salina (T3). A anúria é reflexo da menor perfusão renal e da menor contratibilidade da musculatura lisa vesical. Inadequada perfusão sanguínea também pode gerar aumento na perda de consciência. A diminuição nos movimentos ruminais e surgimento de meteorismo gasoso ruminal, ausência de defecação e reflexo anal, e midríase são decorrentes da menor contratibilidade da musculatura lisa do rúmen, da menor motilidade intestinal e das musculaturas estriadas dos ânus e do músculo retrator da pupila, respectivamente. $\mathrm{O}$ mesmo ocorre com a musculatura esquelética estriada, a qual na fase I se mostra hipercontrátil e na fase seguinte parética ou até em paralisia, levando os animais a pemanecerem em decúbito esternal ou lateral ${ }^{3,14}$. Merece ser citada a presença dos movimentos involuntários da língua e dos lábios. Tais sintomas não são encontrados em quadros clássicos de hipocalcemia natural e são considerados efeitos colaterais provocados pelo $\mathrm{EDTA}^{15}$.

A sintomatologia definitiva de hipocalcemia foi gradativamente desaparecendo no decorrer do tratamento com a solução contendo cálcio. Chamou a atenção a diminuição da taquicardia, voltando aos valores dos batimentos cardíacos do tempo basal, já no início do tratamento. Nesse mesmo período também deixou de ser detectada a hipofonese característica. Outro fato marcante foi a mudança de atitude dos animais, os quais se mostraram bem mais alertas e conscientes. Das 12 fêmeas tratadas, cinco delas se levantaram antes do término do tratamento e as demais em decúbito o fizeram dentro de no máximo 30 minutos após o término da terapia. Concomitantemente, a movimentação ruminal foi restabelecida, assim como o apetite voltou a estar presente após o tratamento em todos os animais. Com o fim da atonia ruminal o meteorismo gasoso também desapareceu, por meio de intensa eructação. A ausência de reflexos pupilar e anal foi completamente revertida.

Em relação às variáveis bioquímicas estudadas, a tabela 3 apresenta os valores médios do cálcio e cálcio livre, a tabela 4 apresenta os resultados de fósforo e magnésio e a tabela 5 apresenta as concentrações da proteína total, albumina e glicose, dos grupos tratado e controle no decorrer do experimento.

Tabela 3 - Valores médios e desvios padrão do cálcio sérico total e do cálcio livre dos bovinos dos grupos tratado e controle nos diferentes tempos - Pirassununga - SP

\begin{tabular}{|c|c|c|c|c|c|c|c|c|c|c|}
\hline \multirow[t]{3}{*}{ Tempos } & \multicolumn{4}{|c|}{ Grupo Tratamento } & \multicolumn{4}{|c|}{ Grupo Controle } & \multirow{3}{*}{$\begin{array}{c}P \\
\mathrm{Ca}\end{array}$} & \multirow{3}{*}{$\begin{array}{c}P \\
\text { Ca livre }\end{array}$} \\
\hline & \multicolumn{2}{|c|}{$\mathrm{Ca}(\mathrm{mMol} / \mathrm{L})$} & \multicolumn{2}{|c|}{$\begin{array}{c}\text { Ca livre } \\
(\mathrm{mMol} / \mathrm{L})\end{array}$} & \multicolumn{2}{|c|}{$\begin{array}{c}\mathrm{Ca} \\
(\mathrm{mMol} / \mathrm{L})\end{array}$} & \multicolumn{2}{|c|}{$\begin{array}{c}\text { Ca Livre } \\
(\mathrm{mMol} / \mathrm{L})\end{array}$} & & \\
\hline & Média & DP & Média & DP & Média & DP & Média & DP & & \\
\hline T 0 & $2,8^{\mathrm{Ba}}$ & 0,2 & $1,2^{\text {в а }}$ & 0,1 & $2,7^{\mathrm{Aa}}$ & 0,2 & $1,2^{\mathrm{Aa}}$ & 0,0 & 0,486 & 0,417 \\
\hline T 1 & $1,9^{\mathrm{Ca}}$ & 0,2 & $0,9^{\mathrm{Ca}}$ & 0,1 & $1,6^{\mathrm{BCb}}$ & 0,2 & $0,7^{\mathrm{Cb}}$ & 0,1 & 0,008 & 0,003 \\
\hline T 2 & $1,9^{\mathrm{Ca}}$ & 0,3 & $0,7^{\mathrm{Ca}}$ & 0,1 & $1,5^{\mathrm{Ca}}$ & 0,2 & $0,6^{\mathrm{Cb}}$ & 0,1 & 0,069 & 0,013 \\
\hline T 3 & $4,1^{\mathrm{Aa}}$ & 0,2 & $1,8^{\mathrm{Aa}}$ & 0,1 & $1,9^{\mathrm{Bb}}$ & 0,3 & $0,8^{\mathrm{B} \mathrm{b}}$ & 0,1 & 0,001 & 0,001 \\
\hline T 4 & $2,8^{\mathrm{Ba}}$ & 0,1 & $1,2^{\mathrm{Ba}}$ & 0,0 & $2,8^{\mathrm{Aa}}$ & 0,1 & $1,2^{\mathrm{Aa}}$ & 0,1 & 0,345 & 0,400 \\
\hline$P$ & \multicolumn{2}{|c|}{0,001} & \multicolumn{2}{|c|}{0,001} & \multicolumn{2}{|c|}{0,001} & \multicolumn{2}{|c|}{0,001} & - & - \\
\hline
\end{tabular}

Letras minúsculas distintas nas linhas indicam diferenças significativas entre os grupos. Letras maiúsculas distintas nas colunas indicam diferenças significativas entre os tempos 
Tabela 4 - Valores médios e desvios padrão do fósforo e magnésio séricos das novilhas dos grupos tratado e controle nos diferentes tempos - Pirassununga - SP

\begin{tabular}{|c|c|c|c|c|c|c|c|c|}
\hline \multirow[t]{3}{*}{ Tempos } & \multicolumn{4}{|c|}{ Grupo Tratamento } & \multicolumn{4}{|c|}{ Grupo Controle } \\
\hline & \multicolumn{2}{|c|}{$\begin{array}{c}\mathbf{P} \\
(\mathrm{mMol} / \mathrm{L})\end{array}$} & \multicolumn{2}{|c|}{$\begin{array}{c}\mathrm{Mg} \\
(\mathrm{mMol} / \mathrm{L}) \\
\end{array}$} & \multicolumn{2}{|c|}{$\begin{array}{c}\mathbf{P} \\
(\mathrm{mMol} / \mathrm{L})\end{array}$} & \multicolumn{2}{|c|}{$\begin{array}{c}\mathrm{Mg} \\
(\mathrm{mMol} / \mathrm{L})\end{array}$} \\
\hline & Média & $\pm \mathrm{DP}$ & Média & $\pm \mathrm{DP}$ & Média & $\pm \mathrm{DP}$ & Média & $\pm \mathrm{DP}$ \\
\hline T 0 & $2,47^{\mathrm{AB}}$ & 0,2 & $0,87^{\mathrm{AB}}$ & 0,08 & $2,72^{\mathrm{A}}$ & 0,2 & $0,91^{\mathrm{A}}$ & 0,12 \\
\hline T 1 & $2,28^{\mathrm{B}}$ & 0,2 & $0,79^{\mathrm{AB}}$ & 0,30 & $2,01^{\mathrm{B}}$ & 0,4 & $0,75^{\mathrm{AB}}$ & 0,15 \\
\hline T 2 & $2,30^{\mathrm{B}}$ & 0,2 & $0,73^{\mathrm{B}}$ & 0,16 & $2,23^{\mathrm{AB}}$ & 0,3 & $0,65^{\mathrm{B}}$ & 0,19 \\
\hline T 3 & $2,37^{\mathrm{AB}}$ & 0,2 & $0,95^{\mathrm{A}}$ & 0,08 & $2,16^{\mathrm{AB}}$ & 0,2 & $0,86^{\mathrm{AB}}$ & 0,16 \\
\hline T 4 & $2,65^{\mathrm{A}}$ & 0,2 & $0,86^{\mathrm{AB}}$ & 0,10 & $2,40^{\mathrm{AB}}$ & 0,3 & $0,90^{\mathrm{A}}$ & 0,08 \\
\hline$P$ & \multicolumn{2}{|c|}{0,012} & \multicolumn{2}{|c|}{0,001} & \multicolumn{2}{|c|}{0,012} & \multicolumn{2}{|c|}{0,048} \\
\hline
\end{tabular}

Letras maiúsculas distintas nas colunas indicam diferenças significativas entre os tempos para cada variável. Não foram observadas diferenças entre grupos para as variáveis $\mathrm{P}$ e $\mathrm{Mg}$

Tabela 5 - Valores médios (M) e desvio padrão da concentração sérica da proteína total e albumina e da glicose plasmática nos grupos tratado e controle no decorrer do experimento - Pirassununga - SP

\begin{tabular}{|c|c|c|c|c|c|c|c|c|c|c|c|c|}
\hline \multirow[t]{3}{*}{ Tempos } & \multicolumn{6}{|c|}{ Grupo Tratamento } & \multicolumn{6}{|c|}{ Grupo Controle } \\
\hline & \multicolumn{2}{|c|}{$\begin{array}{l}\text { Proteína total } \\
\quad(\mathrm{g} / \mathrm{dL})\end{array}$} & \multicolumn{2}{|c|}{$\begin{array}{c}\text { Albumina } \\
(\mathrm{g} / \mathrm{dL})\end{array}$} & \multicolumn{2}{|c|}{$\begin{array}{l}\text { Glicose } \\
(\mathrm{mMol} / \mathrm{L})\end{array}$} & \multicolumn{2}{|c|}{$\begin{array}{l}\text { Proteína total } \\
(\mathrm{g} / \mathrm{dL})\end{array}$} & \multicolumn{2}{|c|}{$\underset{(\mathrm{g} / \mathrm{dL})}{\operatorname{Albumina}}$} & \multicolumn{2}{|c|}{$\begin{array}{c}\text { Glicose } \\
(\mathrm{mMol} / \mathrm{L})\end{array}$} \\
\hline & $\mathbf{M}$ & DP & $\mathbf{M}$ & DP & $\mathbf{M}$ & DP & $\mathbf{M}$ & DP & $\mathbf{M}$ & DP & $\mathbf{M}$ & DP \\
\hline T 0 & 7,29 & 0,53 & 3,02 & 0,13 & $3,7^{\text {в }}$ & 0,4 & 7,24 & 0,66 & 3,02 & 0,39 & $3,6^{\mathrm{B}}$ & 0,3 \\
\hline T 1 & 7,27 & 0,62 & 3,05 & 0,24 & $3,7^{\text {в }}$ & 0,2 & 7,23 & 0,76 & 3,06 & 0,45 & $3,7^{\text {в }}$ & 0,4 \\
\hline T 2 & 7,52 & 0,69 & 3,11 & 0,27 & $4,1^{\text {B }}$ & 0,3 & 7,54 & 1,01 & 3,15 & 0,55 & $4,4^{\mathrm{AB}}$ & 0,5 \\
\hline T 3 & 7,06 & 0,44 & 2,93 & 0,15 & $5,2^{\mathrm{A}}$ & 0,4 & 7,32 & 0,97 & 3,05 & 0,45 & $4,8^{\mathrm{A}}$ & 0,9 \\
\hline T 4 & 7,22 & 0,38 & 3,01 & 0,13 & $3,9^{\mathrm{B}}$ & 0,2 & 7,27 & 0,36 & 3,03 & 0,17 & $4,0^{\mathrm{AB}}$ & 0,3 \\
\hline$P$ & \multicolumn{2}{|c|}{0,638} & \multicolumn{2}{|c|}{0,519} & \multicolumn{2}{|c|}{0,001} & \multicolumn{2}{|c|}{0,971} & \multicolumn{2}{|c|}{0,990} & \multicolumn{2}{|c|}{0,023} \\
\hline
\end{tabular}

Letras minúsculas distintas nas linhas indicam diferenças significativas entre os grupos. Letras maiúsculas distintas nas colunas indicam diferenças significativas entre os tempos

Sem qualquer dúvida, o EDTA gerou nas novilhas uma diminuição temporária da concentração sérica de cálcio total e cálcio livre ou ionizável. Essa diminuição foi detectada, em relação ao momento basal, já quando os animais apresentavam os primeiros sintomas de hipocalcemia (T1). Entretanto, os teores de cálcio total e ionizável se elevaram de forma significativa após o término do tratamento, sendo inclusive mais elevados do que os valores observados no tempo basal.

Em bovinos hígidos cerca de metade do cálcio sérico se apresenta na forma ionizável e a outra remanescente ligada às proteínas séricas. O EDTA após ser infundido no sangue vai aos poucos trocando seus dois cátions $(\mathrm{Na}+)$ por dois átomos de cálcio ionizável, formando com esse elemento uma forte ligação, tornando-o indisponível para o organismo 5 . A velocidade com que o cálcio ionizável declinou foi semelhante à descrita por Mellau, Jorgensen e Enemark ${ }^{5}$ em vacas, ou seja, teve redução contínua e prolongada até o final da infusão. Por outro lado, o tratamento com a solução a base de cálcio elevou de forma rápida e significativa o cálcio livre e total disponibilizando este elemento para o organismo e revertendo os sintomas de hipocalcemia, dentro de 30 minutos, sem que os animais apresentassem posteriormente recidivas deste quadro.

Os teores de fósforo inorgânico dos ruminantes apresentaram diminuições discretas, mas significativas, no decorrer da infusão de EDTA, fato este revertido com o início do tratamento dos animais, o que provocou au- 
mento significativo dos teores desse mineral. Em casos naturais de hipocalcemia da vaca leiteira existe redução natural dos teores séricos de fósforo inorgânico, que pode ser mais intensa que o apresentado experimentalmente ${ }^{4}$. Esta diminuição das concentrações de fósforo constatada durante o experimento sugere que medicamentos que contenham fósforo sejam indicados no tratamento da hipocalcemia em bovinos.

Os teores de magnésio aparentemente não se alteraram no decorrer da infusão de EDTA em relação ao tempo basal. Tais achados também foram encontrados por Mellau, Jorgensen e Enemark ${ }^{5}$. Este fato está associado à baixa afinidade do EDTA ligar-se ao magnésio. Contudo, mesmo sem diferença significativa, percebe-se tendência de redução dos valores séricos de magnésio, especialmente na fase II da hipocalcemia (T2). O tratamento dos animais elevou significativamente os teores séricos de magnésio, que retornaram aos valores basais 24 horas depois de iniciado o experimento.

As concentrações de proteína total e de albumina sérica dos bovinos não se alteraram durante o ensaio clínico. Nos bovinos a glicemia aparentemente pouco se alterou durante a infusão de EDTA. Destaca-se uma tendência de elevação na fase II da hipocalcemia, bem como um aumento significativo no T3 do grupo tratado, devido a solução de cálcio usada no tratamento dos animais conter também glicose. Observou-se também que a glicemia elevada do T2 manteve-se durante a infusão de solução fisiológica nos animais do grupo controle, apresentando valores elevados no T3.

Até onde se conhece o EDTA não interfere no metabolismo da glicose, porém o estresse da manipulação e principalmente a excitação verificada no primeiro estágio clínico dos animais deve ter provocado uma liberação de cortisol, o qual estimula a gliconeogênese com rápida liberação de glicose ${ }^{8}$.

Como não ocorreu redução dos níveis plasmáticos de glicose em nenhum dos grupos durante a indução, o acréscimo desta substância em formulações para o tratamento de quadros de hipocalcemia parece ser desnecessário.

Os resultados obtidos das atividades das enzimas GGT, AST e CK encontram-se na tabela 6. As atividades de GGT dos bovinos não se modificaram no decorrer do ensaio, indicando que não existiram lesões hepáticas decorrentes da indução com EDTA ou tratamento da hipocalcemia. Por outro lado, a atividade de AST se elevou ao término de 24 horas no grupo tratado, em relação aos outros tempos, fato que não ocorreu no grupo controle, provavelmente devido aos elevados desvios padrão observados, pois se verificou elevação numérica da atividade desta enzima neste tempo. Mesmo assim, na $24^{\text {a }}$ hora essa atividade foi semelhante entre o grupo tratado e controle. Tal elevação da atividade de AST está muito mais ligada

Tabela 6 - Valores médios (M) e desvio padrão das atividades da AST, GGT e CK nos grupos tratado e controle no decorrer do experimento - Pirassununga - SP

\begin{tabular}{|c|c|c|c|c|c|c|c|c|c|c|c|c|}
\hline \multirow[t]{3}{*}{ Tempos } & \multicolumn{6}{|c|}{ Grupo Tratamento } & \multicolumn{6}{|c|}{ Grupo Controle } \\
\hline & \multicolumn{2}{|c|}{$\operatorname{AST}(\mathrm{U} / \mathrm{L})$} & \multicolumn{2}{|c|}{ GGT (U/L) } & \multicolumn{2}{|c|}{$\mathrm{CK}(\mathrm{U} / \mathrm{L})$} & \multicolumn{2}{|c|}{ AST (U/L) } & \multicolumn{2}{|c|}{ GGT (U/L) } & \multicolumn{2}{|c|}{$\mathrm{CK}(\mathrm{U} / \mathrm{L})$} \\
\hline & M & DP & M & DP & M & DP & M & DP & M & DP & M & DP \\
\hline T 0 & $50^{\mathrm{B}}$ & 5,3 & 21 & 4,3 & 106 & 59 & 52 & 15,5 & 18 & 3,3 & 152 & 111 \\
\hline T 1 & $53^{\text {в }}$ & 8,5 & 21 & 6,5 & 177 & 105 & 57 & 20,9 & 17 & 2,8 & 161 & 99 \\
\hline T 2 & $61^{\mathrm{AB}}$ & 21,6 & 20 & 5,9 & 192 & 119 & 62 & 24,7 & 18 & 3,2 & 293 & 227 \\
\hline T 3 & $53^{\text {в }}$ & 6,1 & 20 & 5,0 & 230 & 120 & 62 & 25,9 & 18 & 3,7 & 424 & 309 \\
\hline T 4 & $85^{\mathrm{A}}$ & 31,4 & 21 & 5,2 & 363 & 508 & 78 & 27,9 & 19 & 2,5 & 412 & 248 \\
\hline$P$ & \multicolumn{2}{|c|}{0,006} & \multicolumn{2}{|c|}{0,996} & \multicolumn{2}{|c|}{0,416} & \multicolumn{2}{|c|}{0,496} & \multicolumn{2}{|c|}{0,957} & \multicolumn{2}{|c|}{0,157} \\
\hline
\end{tabular}

Letras maiúsculas distintas nas colunas indicam diferenças significativas entre os tempos para cada variável. Não foram observadas diferenças entre grupos para atividades das enzimas estudadas 
às alterações musculares provavelmente oriundas de pequenas lesões desenvolvidas durante o decúbito patológico ou mesmo aos tremores musculares. Este fato pode ser constatado pela elevação numérica, mas não significativa, da atividade da creatina quinase, que apresentou valores acima dos considerados normais para a espécie ${ }^{10}$, sendo a elevação desta ultima enzima indicativa das supracitadas lesões musculares.

\section{Conclusão}

A indução da hipocalcemia com EDTA em bovinos promoveu alterações sintomatológicas significativas além das já conhecidas como: taquicardia acompanhada de hipofonese, atonia ruminal e aumento do tempo de preenchimento capilar, variáveis estas que devem ser consideradas na elaboração do diagnóstico de hipocalcemia.

A elevação concomitante da atividade de AST e CK foi indicativa de lesões musculares durante a

\section{Referências}

1. McDOWELL, L. R. Minerais para ruminantes sob pastejo em regiões tropicais, enfatizando o Brasil. 3. ed. Gainesville: University of Florida, 1999. 92 p.

2. UNDERWOOD, E. J.; SUTTLE, N. F. The mineral nutrition of livestock. 3. ed. Wallingford: Cabi Publishing, 1999. 614 p.

3. RADOSTITS, O. M.; GAY, C. C.; HINCHCLIFF, K. W.; CONSTABLE, P. D. Veterinary medicine. 10. ed. Edimburgo: Saunders Elsevier, 2007. 2156 p.

4. ORTOLANI, E. L. Aspectos clínicos, epidemiológicos e terapêuticos da hipocalcemia de vacas leiteiras. Arquivos Brasileiros de Medicina Veterinária e Zootecnia, v. 47, n. 6, p. 799-808, 1995.

5. MELLAU, L. S. B.; JORGENSEN, R. J.; ENEMARK, J. M. D. Plasma calcium, inorganic phosphate and magnesium during hypocalcaemia induced by a standardized EDTA infusion in cows. Acta Veterinary Scandinavian, v. 42, p. 251-260, 2001.

6. ZIGUER, E. A.; FONTOURA JÚNIOR, J. A. S. D.; CASTILHO, E. M.; MENEZES, L. M.; SEGANFREDO, J. C.; CORRÊA, M. N.; DIONELLO, N. J. L. Impacto econômico produtivo das afecções metabólicas em bovinos de leite no estado Rio Grande do Sul. In: CONGRESSO DE INICIAÇÃO CIENTÍFICA, 14.; ENCONTRO EM PÓS GRADUAÇÃO, 7., Pelotas, 2005. [Anais...], Pelotas: UFPEL, 2005

7. CESCO, F. T. R. S. Efeito terapêutico da Naloxona e do borogluconato de cálcio na reversão da hipocalcemia experimental em vacas. 2006. 82 p. Tese (Doutorado) Faculdade de Ciências Agrárias e Veterinárias da Universidade Estadual Paulista, Jaboticabal, 2006. indução da hipocalcemia por EDTA, sendo importante a inclusão dessas variáveis bioquímicas como auxiliares no diagnóstico da hipocalcemia fisiológica em bovinos.

A diminuição dos níveis séricos de cálcio total e ionizável, magnésio, bem como a discreta diminuição das concentrações de fósforo após a indução da hipocalcemia foi revertida pela administração da solução rica em cálcio, indicando a eficácia desta na recuperação do quadro de hipocalcemia dentro de 30 minutos em bovinos sem apresentar recidivas posteriores.

\section{Agradecimentos}

Os pesquisadores Raimundo A. Barrêto-Júnior e Enrico L. Ortolani são gratos ao $\mathrm{CNPq}$ pelas bolsas de doutorado e produtividade em pesquisa, respectivamente. Antonio H. H. Minervino e Frederico A. M. L. Rodrigues são gratos à FAPESP pelas bolsas de pósgraduação concedidas.

8. SMITH, V. R.; BROWN, W. H. Response of some blood constituents to infusion of disodium ethylenediamine tetraacetate in intact cattle. Journal Dairy Science, v. 46, n. 3, p. 223-226, 1963.

9. FEITOSA, F. L. F. Semiologia Veterinária: a arte do diagnóstico. São Paulo: Rocca, 2004. 824 p.

10.KANEKO, J. J.; HARVEY, J. W.; BRUSS, M. L. Clinical biochemistry of domestic animals. 5. ed. San Diego: Academic Press, 1997. 932 p.

11. BRITO, L. A. B. Avaliação do uso intensivo de cama de frango na alimentação de bovinos: alguns aspectos toxicológicos e do metabolismo do nitrogênio. 1998. 235 p. Tese (Doutorado) - Faculdade de Medicina Veterinária e Zootecnia da Universidade de São Paulo, São Paulo, 1998.

12.SAMPAIO, I. B. M. Estatística aplicada à experimentação animal. 2. ed. Belo Horizonte: Fundação de Estudo e Pesquisa em Medicina Veterinária e Zootecnia, 2002. 265 p.

13. MINITAB. The student edition of MINITAB Statistical software adapted for education. Release 13.0. User's Manual. New York: Addison-Wesley, 2000. CD-ROM.

14. HERDT, T. H. Metabolic diseases of ruminant livestock. The Veterinary Clinics of North America-Food Animal Practice, v. 4, n. 2 , p. $410-437,1988$

15.FENWICK, D. C.; DANIEL, R. C. W. A comparison between the main clinical signs of milk fever in cows with those of hypocalcaemia induced by $\mathrm{NaEDTA}$ solution. Journal of Veterinary Medicine, v. 37, p. 721-728, 1990. 